\title{
Acute Tension Pneumothorax with Extensive Subcutaneous Emphysema in a Post Thoracotomy Child
}

\author{
Dr. See Woan Shiang*, Dr. Simon Jerome Vendargon, Dr. Syed Rasul Bin Ghouse Syed Hamid
}

Departement of Cardiothoracic Surgery, Hospital Sultanah Aminah, Johor Baru, Johor, Malaysia

DOI: $10.36347 /$ sasjs.2020.v06i05.007

| Received: 14.05.2020 | Accepted: 21.05.2020 | Published: 27.05.2020

*Corresponding author: Dr. See Woan Shiang

Abstract

Tension pneumothorax is a life-threatening clinical emergency that requires immediate release of intrathoracic pressure to prevent respiratory failure. Generally, pneumothorax can be caused by physical trauma or lung pathology. Tension pneumothorax after thoracic surgery, even though rare, can pose severe threats to the cardiothoracic surgeon and intensivists during post-operative care. Here, we report a child with empyema thoracis. Thoracotomy and decortication was done. However, the child developed immediate acute tension pneumothorax with large extensive surgical emphysema after chest drain removal. The clinical presentation, intensivist and surgical challenges together with its managements are discussed. To date, there is no similar case reported.

Keywords: Tension pneumothorax, Thoracotomy, Subcutaneous Emphysema, Chest Tube, Empyema.

Copyright @ 2020: This is an open-access article distributed under the terms of the Creative Commons Attribution license which permits unrestricted use, distribution, and reproduction in any medium for non-commercial use (NonCommercial, or CC-BY-NC) provided the original author and source are credited.

\section{INTRODUCTION}

Tension pneumothorax is a life-threatening emergency that requires immediate release of intrathoracic pressure to prevent respiratory failure [1]. It is a progressive build-up of air within the pleural cavity due to lung pathology which forms an 'one-wayvalve'. Generally, pneumothorax can be caused by physical trauma to the chest, lung pathology or as a complication of medical or surgical intervention [2].

\section{CASE PRESENTATION}

A 1 year old girl (weighed $4 \mathrm{~kg}$ ) presented with fever and rapid breathing for one day. She was in respiratory distress dependent on high flow oxygen. Chest X-ray showed a right pleural effusion with collapse consolidation of the right lung.

Computed tomography of thorax discovered a right pleural collection with highest density at lower zone (34-62 Hounsfield Units). The surrounding pleural was thickened and enhanced. Right lower lobe was collapsed and consolidated. Two weeks following antibiotic therapy and chest tube drainage, she remained febrile and oxygen dependent.

A right posterolateral thoracotomy and decortication was performed. The thickened cortex was completely excised from surrounding lung parenchyma with difficulty. Two chest drains size $16 \mathrm{~F}$ were placed.
After surgery, she was ventilated in the paediatric intensive care unit for 1 day with minimal drainage and bubbles from right chest tube. She improved well and extubated on the second day. Right chest tube was removed on her fifth day after surgery. Instantaneously, she developed extensive subcutaneous emphysema in minutes afterwards, involving bilateral chest, anterior, lateral and posterior abdomen (Figure1). She became restless and tachypneic. There was hyper-resonance on percussion over right chest with absent breath sound. Oxygen saturation reduced drastically to $80 \%$.

Tension pneumothorax was diagnosed and the previous right chest drain insertion site purse-string suture was released immediately. A gush of air was heard from the thoracostomy site and the oxygen saturation improved.

Subsequently, a right chest tube size $16 \mathrm{~F}$ was inserted through the previous drain site under aseptic technique, and connected to low pressure suction. Significant bubbling was noted in the drainage bottle. Chest radiograph after the procedure showed significant subcutaneous emphysema, with expanded right lung and right chest tube in situ (Figure-2).

The subcutaneous emphysema improved significantly after the chest tube insertion. 


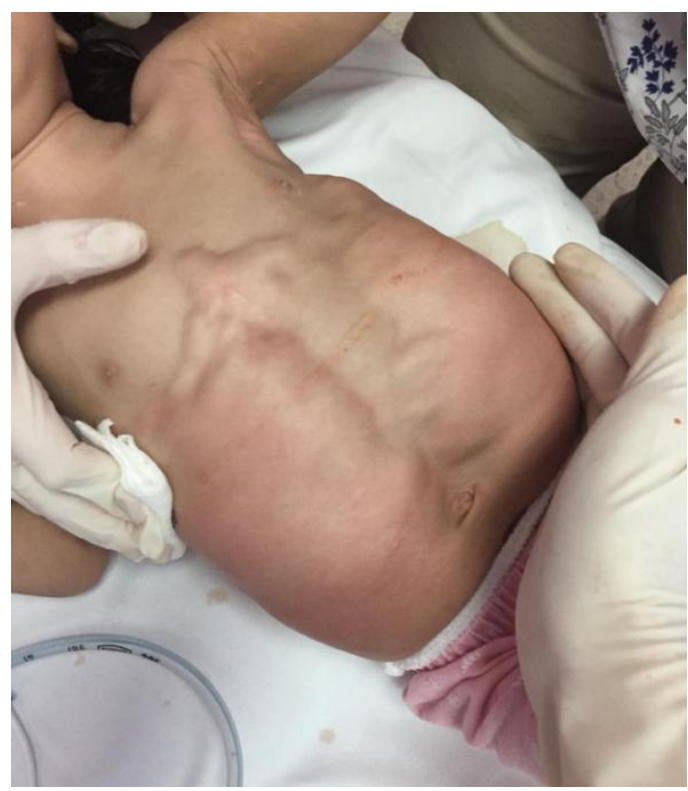

Fig-1: Large massive subcutaneous emphysema
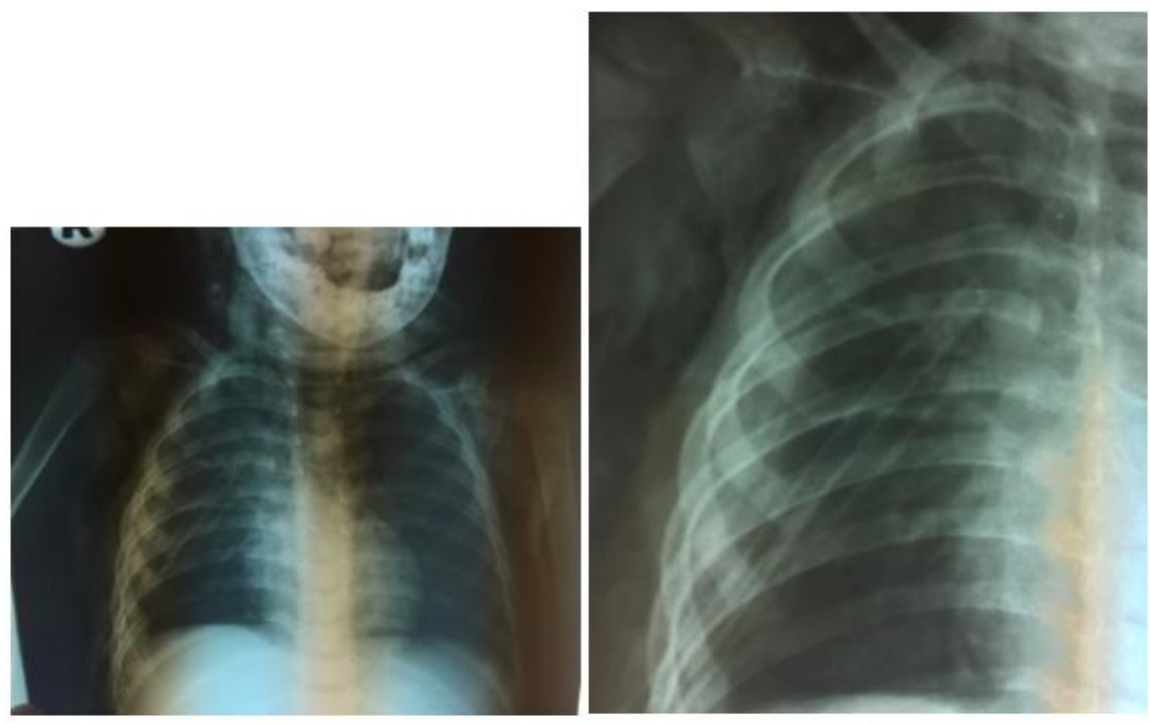

Fig-2: Chest x-ray after chest drain insertion

\section{DISCUSSION}

Tension pneumothorax is a life-threatening clinical emergency that requires immediate release of intrathoracic pressure to prevent respiratory failure. Tension pneumothorax post thoracic surgery, even though rare, can pose a severe threat to the cardiothoracic surgeons and intensivists during postoperative period [3].

During the thoracotomy and decortication surgery, the thickened cortex was completely 'peeled off' from surrounding lung parenchyma with difficulty. The surgery, inevitably, predisposes the child to pneumothorax post operatively due to the thinning of frail localized lung parenchyma.

The child developed tension pneumothorax shortly after removal of the right chest drain. The thinning of lungs parenchyma, together with increased intrathoracic pressure by child's cry, causes rupture of localized thinned lung parenchyma and forms an oneway valve air leak into pleural cavity. Furthermore, the closure of chest drain site traps the pneumothorax and results in acute tension pneumothorax immediately.

In non-surgical patients, needle and tube thoracostomy are the first line management for acute tension pneumothorax. For our case, purse-string suture over the previous chest drain site was cut to open and release the tension pneumothorax instantaneously. The child's respiratory distress resolved significantly with the reduction of intrathoracic pressure.

Once the child is stabilized, Chest tube with size $16 \mathrm{~F}$ is inserted through the thoracostomy wound with aseptic technique and connected to low pressure suctions. Low pressure suction is important for right lung expansion and drainage for massive subcutaneous 
emphysema [4]. Chest radiograph is not mandatory and only done after hemodynamic stabilization and chest tube insertion [5]. It is fundamental in tension pneumothorax management.

Acute tension pneumothorax with extensive subcutaneous emphysema post thoracotomy is rare. In this case, the perpetuating factor to forming subcutaneous emphysema is the child's cry which results in increased intrathoracic pressure. The air escapes from intrapleural space into subcutaneous tissue through the intercostal defect formed by the previous thoracostomy. At the same time, the cutaneous layer of thoracostomy wound is closed with purse-string suture. It prevents the air from escape and thus, the weaker subcutaneous tissues were dissected [6]. In general, subcutaneous emphysema is self-limited. But, in this case, the tension pneumothorax will aggravate the subcutaneous emphysema and the child will progress into severe airway obstruction, respiratory distress and circulatory arrest if no action is taken immediately.

\section{CONCLUSION}

In a child after thoracotomy surgery, it is a great challenge to both the cardiothoracic surgeons and intensivists to identify tension pneumothorax. Although rarely, large subcutaneous emphysema can be the presentation of tension pneumothorax and immediate treatment is mandatory. High index of suspicion for tension pneumothorax is therefore vital and removal of drain site suture will save the patient from respiratory and circulatory arrest.

\section{REFERENCE}

1. Zarogoulidis P, Kioumis I, Pitsiou G, Porpodis K, Lampaki S, Papaiwannou A, Katsikogiannis N, Zaric B, Branislav P, Secen N, Dryllis G. Pneumothorax: from definition to diagnosis and treatment. Journal of thoracic disease. 2014 Oct;6(Suppl 4):S372-6.

2. Kashif S, Gulrez A. Anaesthetic Concerns of Surgical Emphysema Following Thyroidectomy. J Coll Physicians Surg Pak. July 2017;27(3): S4-5.

3. Sengupta S. Post-operative pulmonary complications after thoracotomy. Indian J Anaesth. 2015; 59(9):618-626

4. Tran Q, Mizumoto R, Mehanna D. Management of extensive surgical emphysema with subcutaneous drain: A case report. Int J Surg Case Rep. 2018;44:126-30.

5. Tataroglu O, Erdogan ST, Erdogan MO, Tayfur I, Afacan MA, Yavuz BG, Colak S. Diagnostic Accuracy of InitiaI Chest X-Rays in Thorax Trauma. Journal of the College of Physicians and Surgeons--Pakistan: JCPSP. 2018 Jul 1;28(7):5468.

6. Ema T, Neyatani H, Yamamoto S, Lizuka S, Funai K, Shiiya N. Computed tomography-guided tube thoracostomy for massive subcutaneous emphysema following lung resection: a case report. AME Case Rep. 2019;3:11. 